OPEN ACCESS

Edited by:

Jill Whitall,

University of Maryland, Baltimore,

United States

Reviewed by:

Tao Huang

Shanghai Jiao Tong University, China

David lan Anderson,

San Francisco State University,

United States

Jesper Lundbye-Jensen,

University of Copenhagen, Denmark

*Correspondence:

Rosa Angulo-Barroso

rosa.angulobarroso@csun.edu

Specialty section:

This article was submitted to

Movement Science and Sport

Psychology,

a section of the journal

Frontiers in Psychology

Received: 29 April 2019

Accepted: 15 August 2019

Published: 28 August 2019

Citation:

Angulo-Barroso $R$, Ferrer-Uris $B$

and Busquets A (2019) Enhancing

Children's Motor Memory Retention

Through Acute Intense Exercise:

Effects of Different Exercise Durations.

Front. Psychol. 10:2000.

doi: 10.3389/fpsyg.2019.02000

\section{Enhancing Children's Motor Memory Retention Through Acute Intense Exercise: Effects of Different Exercise Durations}

\author{
Rosa Angulo-Barroso ${ }^{1,2 *}$, Blai Ferrer-Uris ${ }^{1}$ and Albert Busquets ${ }^{1}$ \\ ${ }^{1}$ Institut Nacional d'Educació Física de Catalunya (INEFC), Universitat de Barcelona (UB), Barcelona, Spain, ${ }^{2}$ Department \\ of Kinesiology, California State University, Northridge, CA, United States
}

Physical exercise has been proposed as a viable means to stimulate motor learning. Exercise characteristics, including intensity and duration, may play a role in modulating the exercise effect on motor learning. While some evidence exists regarding the benefits of intense and relatively long exercise, little is known about the effect of short exercise bouts on motor learning, especially in children. This study aimed to assess the effect of long versus short intense exercise bouts on the adaptation and consolidation of a rotational visuomotor adaptation task. The participants were 71 healthy children from two sites divided into three groups: long exercise bout (LONG), short exercise bout (SHORT), and no exercise (CON). Children performed a rotated (clockwise $60^{\circ}$ rotation) motor task on four different occasions: an adaptation set and 1 h, 24 h, and 7 days delayed retention sets. Exercise bouts were performed prior to the adaptation set. Results showed a group effect during motor adaptation $[F(2,68)=3.160 ; p=0.049$; $\left.\eta_{p}^{2}=0.087\right]$, but no statistical differences were found between groups. Regarding retention tests, both exercise groups (LONG and SHORT) showed superior retention compared to CON group $\left[F(2,68)=7.102 ; p=0.002 ; \eta_{p}^{2}=0.175\right]$. No differences were found between exercise groups, indicating similar benefits for the two exercise interventions. Overall, whether the exercise duration was long or short, exercise improved motor memory retention as an estimate of memory consolidation process. The use of short exercise bouts may be suitable to improve children's motor memory consolidation in environments where time constraints exist.

Keywords: children, intense exercise, exercise duration, motor adaptation, motor memory consolidation, initial directional error

\section{INTRODUCTION}

Execution of motor skills involves the integration of perceptual information and motor responses. Learning to adjust a movement to a new mapping between the perceptual and motor components requires a two-phase process, involving adaptation and consolidation (memory formation) of the learned task. During these processes, an initial internal model of the task execution is formed and refined (adaptation) and becomes more stable with the passage of time (consolidation) (Krakauer et al., 2005). Formation of the task's internal model allows for effective use of feedforward 
mechanisms, which in turn facilitate the prediction of the sensory consequences of the movement execution (Krakauer and Shadmehr, 2006). Therefore, internal model formation and feedforward mechanisms are key factors in the effective planning of the required movements to fulfill the task objectives (Contreras-Vidal, 2006).

Recent research suggests that exercise potentially protects motor memory formation from external interferences during consolidation (Rhee et al., 2015) and that adaptation and retention can be enhanced by a single bout of exercise in adults (Taubert et al., 2015) and in children (Lundbye-Jensen et al., 2017; Ferrer-Uris et al., 2018). Previous adult research (Roig et al., 2012; Thomas et al., 2016a,b) proposed that exercise characteristics (e.g., exercise timing, intensity, and duration) play an important role in the modulation of the exercise-induced benefits on motor adaptation and memory consolidation.

Exercise intensity and its timing seem to be important modulators in the exercise-motor learning relation for adults. It has been suggested that moderate intensity exercise presented before the practice of the task facilitates motor adaptation (Statton et al., 2015; Snow et al., 2016). On the other hand, many studies have found improvements in motor memory consolidation when an intense exercise (IE) bout has been presented either before or after the adaptation of the motor task (Roig et al., 2012; Mang et al., 2014, 2016; Ferrer-Uris et al., 2017).

Despite the increasing evidence in adults, little is known about the exercise-motor learning relation in children. To our knowledge, only two studies have addressed exercise effects on children's motor learning. Lundbye-Jensen et al. (2017) found that IE enhanced motor memory consolidation when it was presented after the acquisition of a motor skill, while they did not test the effects of exercise on motor acquisition. Ferrer-Uris et al. (2018) also observed that IE enhanced motor memory consolidation. In addition, they found a stronger effect when exercise was performed immediately before compared to after the motor adaptation presentation. However, Ferrer-Uris et al. (2018) observed that IE had no effect on motor adaptation.

Previous studies have examined how changes in exercise timing or exercise intensity modulate the exercise benefits on motor learning (Roig et al., 2016; Thomas et al., 2016a,b). However, little is known regarding the modulatory effect of exercise duration. In adults, most studies examining the effect of moderate exercise on motor learning have used diverse protocols with durations of $30 \mathrm{~min}$ (Statton et al., 2015; Snow et al., 2016). In contrast, the majority of the studies analyzing the effect of IE have used exercise protocols of shorter durations (between 13 and $15 \mathrm{~min}$ of interval exercise) (Roig et al., 2012; Mang et al., 2014; Ferrer-Uris et al., 2017). To the best of our knowledge, no study has assessed the effect of an IE bout shorter than $12 \mathrm{~min}$, or the modulatory effect of different exercise durations on motor learning in adults, and much less in a pediatric population.

Unlike the literature void regarding motor learning, previous research has studied the effect of short exercise bouts on cognitive learning, which may guide predictions in the motor learning domain. In adults, two 3-min sprints enhanced the acquisition and retention (exploratory comparisons) of a novel vocabulary task (Winter et al., 2007). In children, Etnier et al. (2014) observed improvements in a novel vocabulary task and its recall after performing a graded maximal running test lasting $9.88 \mathrm{~min}$ on average (ranging between 6.42 and $20 \mathrm{~min}$ ). Moreover, some school-based exercise strategies of $10 \mathrm{~min}$ or less have obtained positive effects on children's attention (Mahar et al., 2006; Ma et al., 2014). Therefore, short exercise bouts could show some promising results in enhancing motor learning.

Here, we investigated the effect of two IE intervention durations (short IE: a short bout of $5 \mathrm{~min}$, and long IE: a long bout of $13 \mathrm{~min}$ ) on the adaptation and retention of a motor task, which was a rotational visuomotor adaptation task (rVMA). We hypothesized that (1) IE would not enhance the adaptation of the rVMA, (2) the long IE would enhance consolidation of the rVMA, and (3) the short IE would also improve consolidation of the rVMA, although presenting a smaller effect compared to the long IE.

\section{MATERIALS AND METHODS}

Seventy-one children ( 40 male and 31 female, $9.13 \pm 0.8$ years) were recruited from two schools, one located in Barcelona, Spain (Site 1) and another in Los Angeles, United States (Site 2) (Table 1). Participants were excluded when they presented one or more of the following attributes: prior experience with the rVMA; left-handedness; low physical activity engagement; body mass index (BMI) above obesity threshold; intellectual quotient (IQ) below average score; parent-reported history of neurological, psychiatric, and/or physical impairment; non-corrected 20/20 vision; or current intake of medications affecting the nervous system and/or the ability to learn. By design, participants at Site 2 were randomly assigned to the experimental groups: (LONG) participants exercised for 13 min before practicing the rVMA; (SHORT) participants exercised for 5 min before practicing the rVMA; and (CON) who rested before engaging in the rVMA. At Site 1, participants were assigned to LONG or CON. Participants' random distribution was separately checked at each site using age and fitness level as stratification factors because it has been reported that these two factors may impact the exercise effect on cognitive performance (Labelle et al., 2013), as well as the development of visuomotor representations (Contreras-Vidal et al., 2005). Identical materials and data collection and reduction procedures were used in both sites. However, group distribution was different by design.

Written and informed consent from all participants' guardians and written assent from participants were provided prior to initiating the study in accordance with the Declaration of Helsinki. The study was approved by the Ethics Committee of Clinic Researches of the Catalan Sport Administration, and the Committee for the Protection of Human Subjects of the California State University, Northridge, CA, United States.

\section{Procedure}

A total of four sessions were completed by each participant (Figure 1). Prior to the start of the first study session, guardians and children completed the Physical Activity Questionnaire for Children (PAQ-C) (Kowalski et al., 1997; 
TABLE 1 | Group characteristics.

\begin{tabular}{|c|c|c|c|c|c|c|c|}
\hline & \multicolumn{3}{|c|}{ LONG } & \multirow{2}{*}{$\begin{array}{c}\text { SHORT } \\
\text { Site } 2\end{array}$} & \multicolumn{3}{|c|}{ CON } \\
\hline & Site 1 & Site 2 & Site $1+2$ & & Site 1 & Site 2 & Site $1+2$ \\
\hline N & 10 & 17 & 27 & 19 & 11 & 14 & 25 \\
\hline Sex (male/female) & $7 / 3$ & $8 / 9$ & $15 / 12$ & $11 / 8$ & $6 / 5$ & $9 / 5$ & $15 / 10$ \\
\hline Age (years) & $9.16 \pm 1.1$ & $9.28 \pm 0.7$ & $9.24 \pm 0.9$ & $9.19 \pm 0.9$ & $8.78 \pm 0.7$ & $9.10 \pm 0.6$ & $8.96 \pm 0.7$ \\
\hline Height (cm) & $135.66 \pm 8.7$ & $138.06 \pm 7.2$ & $137.17 \pm 7.7$ & $135.97 \pm 7.6$ & $135.45 \pm 6.4$ & $132.51 \pm 5.1$ & $133.80 \pm 5.8$ \\
\hline Body mass (kg) & $32.90 \pm 7.3$ & $32.61 \pm 6.8$ & $32.71 \pm 6.9$ & $34.85 \pm 8.5$ & $32.82 \pm 8.0$ & $29.78 \pm 0.7$ & $31.11 \pm 7.4$ \\
\hline $\mathrm{BMI}\left(\mathrm{kg} / \mathrm{m}^{2}\right)$ & $17.72 \pm 2.5$ & $17.04 \pm 3.0$ & $17.29 \pm 2.8$ & $18.65 \pm 3.2$ & $17.67 \pm 2.6$ & $16.90 \pm 3.5$ & $17.24 \pm 3.1$ \\
\hline Estimated $\mathrm{VO}_{2} \max (\mathrm{ml} / \mathrm{kg} / \mathrm{min})$ & $50.90 \pm 4.2$ & $46.16 \pm 2.0$ & $47.91 \pm 3.7$ & $44.80 \pm 2.9$ & $51.19 \pm 5.6$ & $45.36 \pm 2.9$ & $47.93 \pm 5.1$ \\
\hline
\end{tabular}

LONG, long exercise bout group; SHORT, short exercise group; CON, no exercise group; BMI, body mass index.

Benítez-Porres et al., 2016) and the Physical Activity Readiness Questionnaire for children.

In Session 1, basic anthropometric characteristics (height and weight), IQ, and fitness level (estimated $\mathrm{VO}_{2} \max$ ) of the children were assessed. IQ was assessed using the latest available version of the Test of Non-verbal Intelligence (TONI) (version 4 for the American sample and version 2 for the Spanish sample). Fitness level was evaluated using the 20 -m shuttle-run test (20mSRT) (Léger et al., 1988). Beat-by-beat values for the RR intervals were collected with a Polar RS800CX (Polar Electro) at $1000 \mathrm{~Hz}$ frequency. A young fit adult accompanied the children running beside them to help maintain the pace and provide encouragement. Additionally, children carried a pictured card from side to side of the field in every even-numbered lap. The collected cards were used in a memory game at the end of Session 2 (only as incentive).

Forty-eight hours after Session 1, participants started Session 2 by being familiarized ( 20 trials) with the rVMA without cursor rotation $\left(0^{\circ}\right)$. Following familiarization, a baseline set (104 trials)

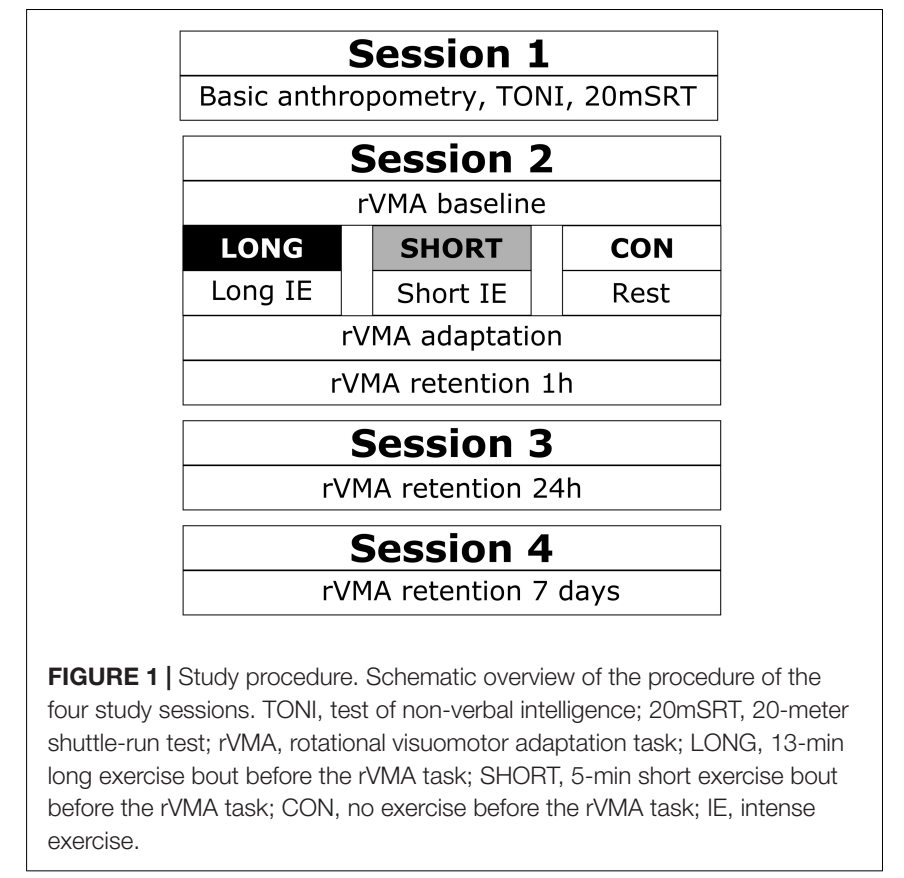

was conducted to assess initial rVMA performance without cursor rotation $\left(0^{\circ}\right)$. Afterward, participants in both exercise groups engaged in the IE. To ensure equal test timing among groups between baseline and the next rVMA set, SHORT rested for $8 \mathrm{~min}$ before the IE warm-up. The transition time between the IE and the next rVMA set was 4 min for both exercise groups. Participants in CON had a rest period of $25 \mathrm{~min}$ while other participants exercised. After the IE or the rest period, participants engaged in the rVMA adaptation set ( $\mathrm{AD}, 312$ trials) where a $60^{\circ}$ clockwise rotation was applied to the cursor movement. After a 1-h rest, all participants performed a second set of the $60^{\circ}$ rotated rVMA. This was the first retention set (RT1h, 104 trials).

At the end of Session 2, all participants played the memory game with the cards they collected during the 20mSRT and the IE. During rest periods, participants were allowed to read or hold a conversation and were prevented from performing additional exercise or musical activity. During the third and fourth sessions, participants performed an additional set of the $60^{\circ}$ rotated rVMA, $24 \mathrm{~h}$ and 7 days (RT24h and RT7d, 104 trials each) after the $\mathrm{AD}$ set.

\section{Rotational Visuomotor Adaptation Task}

The rVMA task was conducted in a quiet room, where participants were seated $1 \mathrm{~m}$ away from a 19-inch screen. Participants controlled a screen cursor using a joystick. Cursor movement coordinates were registered at $120 \mathrm{~Hz}$ using an A/D NI-6008 card (National Instruments Corporation). A new target randomly appeared every $2 \mathrm{~s}$ in one of eight possible positions. Participants were instructed to run the cursor over the target doing one single move "as fast and as straight as possible" and then to move back to the home position (screen center).

\section{Intense Exercise}

Two different IE protocols were programed: long IE and short IE. Both IE procedures were similar to a $20 \mathrm{mSRT}$ but with specific running intensities and volumes. During both exercise bouts, two speeds based on a percentage of the estimated $\mathrm{VO}_{2}$ max were combined: a fast-paced speed $\left(85 \%\right.$ of $\left.\mathrm{VO}_{2} \max \right)$ and a slow-paced speed $\left(60 \% \mathrm{VO}_{2} \mathrm{max}\right)$. The long IE (13 min total) interspersed three series of $3 \mathrm{~min}$ of fast speed with two series of $2 \mathrm{~min}$ of slow speed. The short IE ( 5 min total) consisted of two series of $2 \mathrm{~min}$ of fast speed separated by $1 \mathrm{~min}$ of slow speed. Before the 
start of the IE, participants of both exercise groups underwent a warm-up protocol consisting of 2 min running at slow speed and $1 \mathrm{~min}$ of fast speed. The warm-up was used to familiarize the participants with the running speeds of the IE. A 5-min rest period was defined between the warm-up and the IE. Participants' heart rate was captured following the same procedure described in the $20 \mathrm{mSRT}$. Heart rate data for the $85 \%$ of $\mathrm{VO}_{2}$ max series (Table 2) indicated similar intensities across exercise groups. To encourage participants' effort, an adult also accompanied them during the exercise, and they continued collecting memory game cards during the IE.

\section{Data Reduction}

Custom MATLAB R2014b programs (The MathWorks, Inc.) were used to reduce the Cartesian positions of the joystick movement. Data were low-pass filtered using an eight-order dual-pass Butterworth filter with a cut-off frequency of $12 \mathrm{~Hz}$. Accepted trials fulfilled the following conditions: startup position found within $20 \%$ of the target radius (distance from center to target), and total traveled distance from the screen center to the end of the movement was equal to or greater than $90 \%$ of the target radius. For the accepted trials, the nearest point to an outward movement equal to $10 \%$ of the target radius distance was defined as the movement onset. Movement offset was defined as the point where the speed of the movement decreased to $10 \%$ of its maximum value. The $\mathrm{AD}$ set was divided in epochs of eight trials for further analyses.

\section{Variables}

Movement descriptive characteristics were: movement time (MT, $\mathrm{ms}$ ), travel distance (TD, $\mathrm{cm}$ ), and reaction time (RT, ms). MT and TD were defined as the time and the displacement, respectively, between movement onset and offset. RT was defined as the time window from the target appearance to the movement onset. The initial directional error (IDE, degree) was computed as a representative of output error (Figure 2). IDE was calculated as the angular difference between two linear vectors, representing the ideal movement trajectory and the real initial trajectory from the home position to the cursor position at $80 \mathrm{~ms}$ after movement onset. IDE was used as a measure of the rotation adaptation or motor memory consolidation, avoiding the possible trajectory correction through perceptual feedback (Contreras-Vidal et al., 2005). IDE is considered a good estimate of movement planning and, thus, of the formation of the internal model of the motor skill (Contreras-Vidal et al., 2005; King et al., 2009; Kagerer and Clark, 2014). Moreover, this variable was utilized as our main outcome because IDE has been observed to be particularly sensitive to

TABLE 2 | Group average and standard deviation heart rate data of the last 1/6th of each $85 \% \mathrm{VO}_{2}$ max series.

\begin{tabular}{lcccc}
\hline & Serie 1 $\mathbf{( b p m )}$ & Serie 2 $\mathbf{( b p m )}$ & Serie 3 $(\mathbf{b p m})$ & Grand mean \\
\hline LONG & $185.0 \pm 13.8$ & $189.5 \pm 14.4$ & $190.0 \pm 17.7$ & $188.4 \pm 13.8$ \\
SHORT & $183.5 \pm 20.3$ & $186.2 \pm 19.0$ & NA & $184.8 \pm 19.5$
\end{tabular}

T-test of the 85\% VO $\mathrm{VO}_{2}$ max. Grand mean: $t_{(42)}=0.695, p=0.491$. LONG, long exercise bout group; SHORT, short exercise group; bpm, beats per minute.

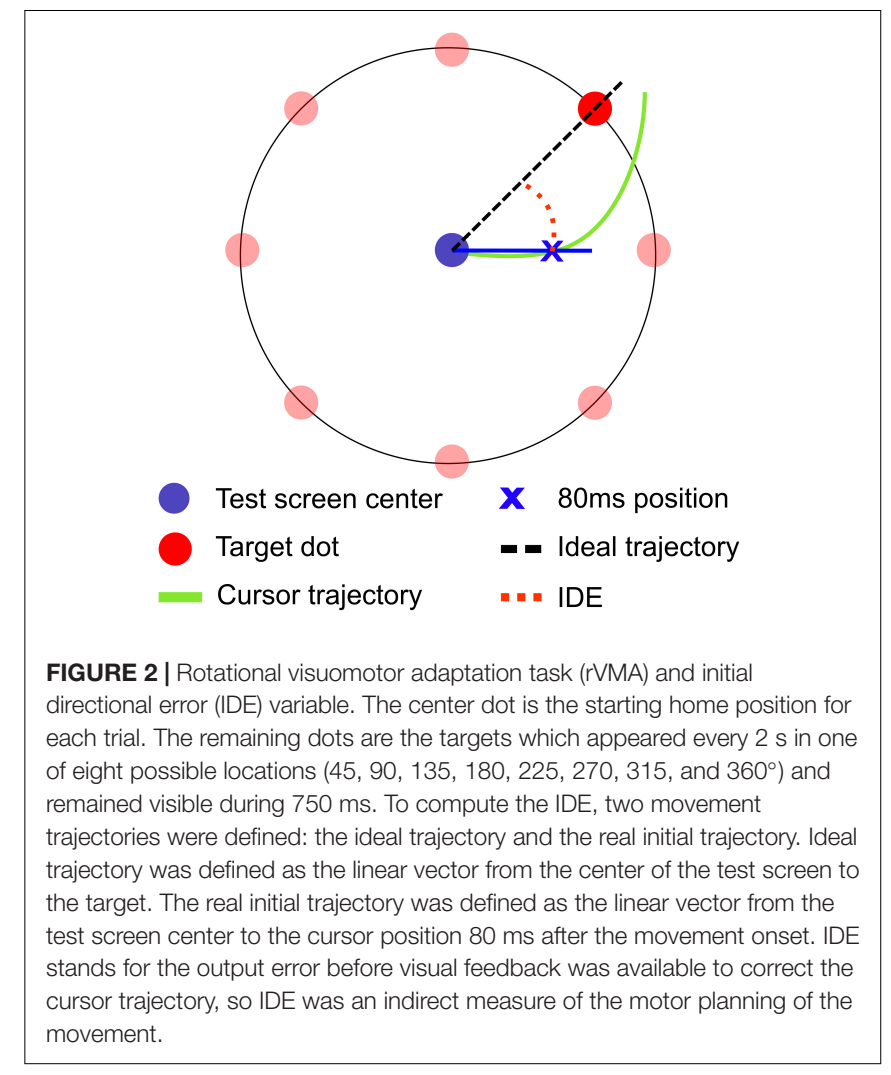

exercise effects (Ferrer-Uris et al., 2017, 2018). Considering the large inter-subject variability usually presented by children, computed adaptation and retention variables were normalized by the participant's baseline mean values.

Initial directional error followed a decay flow during $\mathrm{AD}$ set that resulted to be best fitted by a double exponential function, as seen in previous research (Krakauer et al., 2005). The error decay presented an initial rapid reduction followed by a slower decay of the error. The initial rate of learning $(\mathrm{RL})$ is a measure of the initial rapid error reduction. Individual RL was computed for IDE (RL-IDE) as the first derivative of the first half of the double exponential function and evaluated at epoch 1 , similarly as described in Coats et al. (2014).

\section{Data Analysis}

Normality distribution was checked for all variables via histograms and the Kolmogorov-Smirnov's normality test. Variable transformation or non-parametric alternative tests were used when necessary (baseline MT and baseline IDE were transformed). To control for the group distribution differences across sites, we implemented a four-step process: (1) possible site differences at baseline were explored through Student's $t$-test or the Mann-Whitney $U$ test comparing age, estimated $\mathrm{VO}_{2} \max$, and the rVMA baseline variables (MT, TD, RT, and IDE); (2) to examine possible group differences at baseline, age, and estimated $\mathrm{VO}_{2}$ max and rVMA baseline variables were explored through one-way analysis of variance (ANOVA); (3) we studied the association between the IDE variable and those variables that 
presented baseline site or group differences, using the Pearson correlation coefficient; and (4) those variables that presented significant site or group differences and significant correlation with the IDE were used as covariates in the subsequent analyses. For the first study hypothesis, where the effect of the IE bout on the $\mathrm{AD}$ of the rVMA task was examined, mean values of the AD error variables (IDE and RL-IDE) were compared using one-way analysis of covariance (ANCOVA) with group as the independent factor. The second and third hypotheses, related to the effect of exercise on motor retention and the modulation effect of the exercise length on the retentions of the $\mathrm{rVMA}$, were explored with two-way (Group $\times$ Set) repeated measures ANCOVAs, comparing the mean IDE retention values. Greenhouse-Geisser sphericity-corrected values were reported when appropriate. Bonferroni-corrected post hoc tests were performed when significant differences were found.

The effect size of the different tests was calculated according to Cohen (1988): $d$ for $t$-test (0.2 small, 0.5 medium, and 0.8 large effect), $r$ for Mann-Whitney $U$ test ( 0.1 small, 0.3 medium, and 0.5 large effect), and $\eta_{\mathrm{p}}^{2}$ for ANOVAs and ANCOVAs (0.01 small, 0.06 medium, and 0.14 large effect). Statistical significance was set at $p<0.05$ for all comparisons.

\section{RESULTS}

The analysis of the possible site effect on the participants' characteristics showed that participants from Site 1 (BCN) presented higher $\mathrm{VO}_{2} \max \left[t_{(69)}=5.026 ; p<0.001 ; d=1.442\right]$, compared to the participants in Site 2 (LA). When the site effect was explored for the rVMA baseline variables, we found significant statistical differences between sites for the MT $\left[t_{(69)}=8.331 ; p<0.001 ; d=2.164\right]$, TD $\left[t_{(69)}=6.496 ; p<0.001\right.$; $d=1.786]$, and RT $\left[t_{(69)}=2.979 ; p<0.001 ; d=0.805\right]$ variables, indicating that participants from Site 1 executed the task with longer MT, and shorter TD and RT. However, IDE, which does not directly depend on the movement approach but on the planning of the movement, showed no differences between sites $\left[t_{(69)}=0.342 ; p=0.733 ; d=0.160\right]$.

Participants' characteristics and the average rVMA baseline performance were analyzed via ANOVAs to check main group effects. No group differences were found regarding participant characteristics (age and $\mathrm{VO}_{2} \max$ ). Similarly, no group differences were observed regarding rVMA baseline performance (MT, TD, RT, and IDE; see Table 3 for means and $\mathrm{SD})$. Therefore, groups presented a balanced distribution in terms of age, $\mathrm{VO}_{2} \mathrm{max}$, and rVMA baselines performance despite the aforementioned site effects.

Correlation analysis between IDE and those variables presenting site differences at baseline (MT, TD, RT, and estimated VO2max) was performed using the Pearson correlation coefficient. IDE presented low but significant correlations with the MT $(r=0.255 ; p=0.032)$ and the RT $(r=-0.411 ; p<0.001)$ variables. Therefore, baseline MT and RT were used as covariates in the subsequent analyses.

To examine the possible exercise effect on the adaptation of the rVMA task, we explored the effect of the SHORT, LONG, and CON interventions on the average IDE of the adaptation set, as well as the initial RL of the IDE variable (RL-IDE) (Figure 3). The ANCOVA revealed group differences regarding $\operatorname{IDE}\left[F_{(2,68)}=3.160 ; p=0.049 ; \eta_{\mathrm{p}}^{2}=0.087\right]$ (Figure 4). However, no statistical differences were found between groups. Moreover, no significant group differences or covariate effects were found for RL-IDE. In addition, the covariate MT, but not RT, was found to be significant in the IDE analysis $\left[F_{(2,68)}=7.1600\right.$; $\left.p=0.009 ; \eta_{\mathrm{p}}^{2}=0.098\right]$.

The effects of the IEs on the retention sets of the rVMA task were analyzed using a repeated measure ANCOVA. A significant group effect was found for the IDE $\left[F_{(2,68)}=6.108 ; p=0.004\right.$; $\left.\eta_{\mathrm{p}}^{2}=0.156\right]$. Post hoc analysis indicated that both exercise groups had lower IDE values in comparison to CON (LONG: $p=0.002$; $d=0.868$, SHORT: $p=0.022 ; d=0.708$ ) (Figure 5). No IDE difference was observed between the two exercise groups (SHORT and LONG). Furthermore, no covariate turned out to be significant. In addition, no significant main set effect or Group $\times$ Set interaction effect were observed.

\section{DISCUSSION}

The main focus of this study was to observe the effect of two exercise protocol durations ( $5 \mathrm{~min}$, short bout, and $13 \mathrm{~min}$, long bout) on the adaptation and retention of a rVMA using IDE as the main outcome variable.

Regarding motor adaptation, previous research suggested that IE may produce a null effect on adaptation in adults (FerrerUris et al., 2017) and children (Ferrer-Uris et al., 2018), possibly because of inducement of fatigue (Roig et al., 2012) or excessive arousal (McMorris and Hale, 2012). We hypothesized that both long and short IE would not affect motor adaptation. According to our hypothesis, all groups presented similar performance during the adaptation set. The results of the present study add new evidence and support the previous evidence about the exercise-induced effects on motor learning, suggesting that IE may not improve motor adaptation even when exercise duration is short. However, more research may be necessary to further speculate on this topic, especially considering that a main group effect during adaptation was found, though it could not be confirmed during the pairwise group comparisons. More studies focusing on the analysis of the underlying mechanisms regarding exercise-induced benefits under different exercise intensity and duration conditions, both in adults and children, are needed. Furthermore, our results should be interpreted with caution considering the asymmetrical group distribution in this study.

Regarding motor retention (as an estimate of the motor memory consolidation process) and based on previous research using similar long exercise bouts to enhance motor memory consolidation in adults (Roig et al., 2012; Ferrer-Uris et al., 2017) and children (Lundbye-Jensen et al., 2017; Ferrer-Uris et al., 2018), we hypothesized that LONG would enhance motor memory consolidation. Furthermore, based on the evidence that intense short exercise bouts have facilitated declarative learning in adults (Winter et al., 2007) and children (Etnier et al., 2014), we hypothesized that, although LONG would present a greater 
TABLE 3 | Mean and SD performance values on the rotational visuomotor adaptation task (rVMA) for each group, site, and set.

\begin{tabular}{|c|c|c|c|c|c|c|c|}
\hline \multirow[b]{2}{*}{ Sets } & \multicolumn{3}{|c|}{ LONG } & \multirow{2}{*}{$\begin{array}{c}\text { SHORT } \\
\text { Site } 2\end{array}$} & \multicolumn{3}{|c|}{ CON } \\
\hline & Site 1 & Site 2 & Site $1+2$ & & Site 1 & Site 2 & Site $1+2$ \\
\hline \multicolumn{8}{|l|}{ Baseline } \\
\hline $\mathrm{MT}(\mathrm{ms})$ & $196.15 \pm 28.64$ & $150.36 \pm 23.47$ & $167.32 \pm 33.62$ & $145.72 \pm 14.71$ & $195.72 \pm 26.41$ & $143.61 \pm 13.70$ & $166.53 \pm 33.00$ \\
\hline $\mathrm{TD}(\mathrm{cm})$ & $6.85 \pm 0.32$ & $7.57 \pm 0.45$ & $7.30 \pm 0.53$ & $7.35 \pm 0.46$ & $6.67 \pm 0.36$ & $7.53 \pm 0.46$ & $7.15 \pm 0.59$ \\
\hline $\mathrm{RT}(\mathrm{ms})$ & $404.15 \pm 36.97$ & $439.85 \pm 37.90$ & $426.63 \pm 40.81$ & $433.24 \pm 46.66$ & $419.14 \pm 32.54$ & $459.93 \pm 37.16$ & $441.98 \pm 40.20$ \\
\hline IDE (deg) & $12.44 \pm 6.08$ & $10.40 \pm 2.36$ & $11.15 \pm 4.15$ & $11.76 \pm 6.23$ & $10.67 \pm 3.26$ & $9.96 \pm 2.10$ & $10.27 \pm 2.63$ \\
\hline \multicolumn{8}{|c|}{ Adaptation } \\
\hline $\mathrm{MT}(\mathrm{ms})$ & $199.04 \pm 26.7$ & $145.96 \pm 20.36$ & $165.62 \pm 34.41$ & $146.00 \pm 18.38$ & $214.39 \pm 38.35$ & $151.75 \pm 17.52$ & $179.31 \pm 42.26$ \\
\hline $\mathrm{TD}(\mathrm{cm})$ & $7.00 \pm 0.31$ & $7.75 \pm 0.38$ & $7.74 \pm 0.51$ & $7.52 \pm 0.45$ & $6.83 \pm 0.27$ & $7.79 \pm 0.18$ & $7.37 \pm 0.53$ \\
\hline $\mathrm{RT}(\mathrm{ms})$ & $408.23 \pm 46.82$ & $455.30 \pm 38.84$ & $437.87 \pm 47.16$ & $451.73 \pm 47.45$ & $423.11 \pm 45.03$ & $456.94 \pm 32.60$ & $442.06 \pm 41.41$ \\
\hline IDE (deg) & $28.67 \pm 6.01$ & $30.44 \pm 8.74$ & $29.78 \pm 7.77$ & $30.08 \pm 7.62$ & $32.38 \pm 7.94$ & $32.81 \pm 10.18$ & $32.62 \pm 9.08$ \\
\hline RL_IDE & $-4.09 \pm 4.65$ & $-5.16 \pm 3.29$ & $-4.77 \pm 3.77$ & $-6.11 \pm 6.29$ & $-6.10 \pm 5.74$ & $-4.29 \pm 2.19$ & $-5.10 \pm 4.12$ \\
\hline \multicolumn{8}{|c|}{ Retention $1 \mathrm{~h}$} \\
\hline $\mathrm{MT}(\mathrm{ms})$ & $204.79 \pm 30.48$ & $142.57 \pm 16.32$ & $165.61 \pm 37.72$ & $133.75 \pm 13.61$ & $193.51 \pm 31.14$ & $140.47 \pm 19.54$ & $163.81 \pm 36.51$ \\
\hline TD (cm) & $7.06 \pm 0.34$ & $7.91 \pm 0.32$ & $7.60 \pm 0.53$ & $7.91 \pm 0.42$ & $7.00 \pm 0.31$ & $7.90 \pm 0.29$ & $7.50 \pm 0.54$ \\
\hline $\mathrm{RT}(\mathrm{ms})$ & $418.07 \pm 45.40$ & $448.73 \pm 43.72$ & $437.38 \pm 46.01$ & $443.98 \pm 53.11$ & $432.09 \pm 41.01$ & $451.63 \pm 35.82$ & $443.03 \pm 38.65$ \\
\hline IDE (deg) & $17.43 \pm 3.40$ & $18.73 \pm 6.14$ & $18.25 \pm 5.26$ & $20.16 \pm 7.01$ & $24.04 \pm 10.14$ & $22.35 \pm 7.00$ & $23.09 \pm 8.37$ \\
\hline \multicolumn{8}{|c|}{ Retention 24 h } \\
\hline $\mathrm{MT}(\mathrm{ms})$ & $211.88 \pm 33.80$ & $143.97 \pm 17.56$ & $169.12 \pm 41.25$ & $137.37 \pm 16.46$ & $194.72 \pm 31.35$ & $143.77 \pm 22.78$ & $166.19 \pm 36.84$ \\
\hline $\mathrm{TD}(\mathrm{cm})$ & $7.16 \pm 0.35$ & $7.82 \pm 0.27$ & $7.58 \pm 0.44$ & $7.89 \pm 0.37$ & $7.27 \pm 0.45$ & $7.75 \pm 0.29$ & $7.54 \pm 0.44$ \\
\hline $\mathrm{RT}(\mathrm{ms})$ & $414.44 \pm 48.04$ & $459.42 \pm 43.60$ & $442.76 \pm 49.58$ & $444.00 \pm 51.22$ & $427.44 \pm 30.95$ & $450.71 \pm 41.64$ & $440.47 \pm 38.44$ \\
\hline IDE (deg) & $17.02 \pm 4.28$ & $18.94 \pm 5.40$ & $18.23 \pm 5.02$ & $18.76 \pm 5.71$ & $23.49 \pm 7.42$ & $20.85 \pm 4.28$ & $22.01 \pm 5.89$ \\
\hline \multicolumn{8}{|c|}{ Retention 7 days } \\
\hline $\mathrm{MT}(\mathrm{ms})$ & $203.87 \pm 31.36$ & $144.63 \pm 20.02$ & $166.57 \pm 37.91$ & $133.67 \pm 16.12$ & $195.37 \pm 33.96$ & $137.12 \pm 11.90$ & $162.75 \pm 37.79$ \\
\hline $\mathrm{TD}(\mathrm{cm})$ & $7.30 \pm 0.31$ & $7.81 \pm 0.39$ & $7.62 \pm 0.44$ & $7.79 \pm 0.32$ & $7.27 \pm 0.34$ & $7.77 \pm 0.35$ & $7.55 \pm 0.42$ \\
\hline $\mathrm{RT}(\mathrm{ms})$ & $406.93 \pm 51.39$ & $445.37 \pm 44.39$ & $431.14 \pm 49.85$ & $431.25 \pm 46.36$ & $422.40 \pm 42.6$ & $443.42 \pm 30.53$ & $434.17 \pm 37.07$ \\
\hline IDE (deg) & $17.72 \pm 4.41$ & $17.20 \pm 5.59$ & $17.39 \pm 5.10$ & $19.17 \pm 5.07$ & $21.32 \pm 7.90$ & $20.97 \pm 6.20$ & $21.12 \pm 6.84$ \\
\hline
\end{tabular}

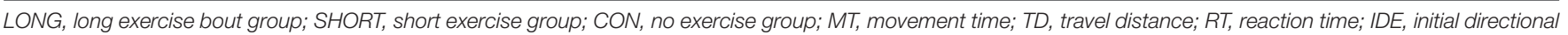
error; $R L$, rate of learning.

effect, SHORT would also improve motor memory consolidation. Results indicated that the movement planning of the motor skill (IDE) improved during the retention sets presumably as a result of the exercise interventions. Interestingly, this effect seemed to take place already $1 \mathrm{~h}$ from adaptation and lasted for 7 days. However, further research would be necessary to examine if motor learning form (e.g., motor adaption or motor sequence learning) may impact the time course of the motor consolidation process. To the best of our knowledge, this is the first piece of evidence of a facilitation of motor memory consolidation after a bout of exercise as short as 5-min duration. These exercisebased improvements cannot be solely explained by a site effect since differences were not found between sites for the IDE variable at baseline and LONG was composed by participants from both sites. In addition, when looking at the movement descriptive variables (Table 3), it appears that groups presented a similar movement approach during retentions, which indicates that IDE differences among groups may not be explained by the movement approach, but by the participants' movement planning. Moreover, IDE group differences during retention were not related to any of the controlled variables.

Previous research in adult populations has proposed that exercise benefits on consolidation of declarative memory
(Winter et al., 2007; Segal et al., 2012) and motor memory (Mang et al., 2014; Skriver et al., 2014) may be produced by a stimulation of the learning-related neuroplasticity mechanisms. Neurotrophins, such as brain-derived neurotrophic factor, and catecholamines (epinephrine, norepinephrine, and dopamine), are some of the biomarkers that are usually associated with enhancements in synaptic plasticity (Taubert et al., 2015). Intense physical exercise has been related to increases in peripheral blood concentrations of these neurochemical compounds (Winter et al., 2007). Increases in blood lactate concentration have also been seen to positively correlate with improvements in motor memory consolidation (Skriver et al., 2014). Even though a causal relation between lactate and synaptic plasticity may be difficult to ascertain, it is thought that lactate could act as intermediary in the synaptic plasticity mechanisms, possibly involved in growth-factor signaling and brain energetics (Skriver et al., 2014; Taubert et al., 2015). However, these relations have to be interpreted with caution because of the difficulties of measuring central concentration of these biomarkers in humans, thereby limiting the establishment of a causal link between exercise, neurochemicals, and consolidation. Moreover, despite some evidence regarding exercise-induced catecholamine increases (Wigal et al., 2003), little is known of these mechanisms 


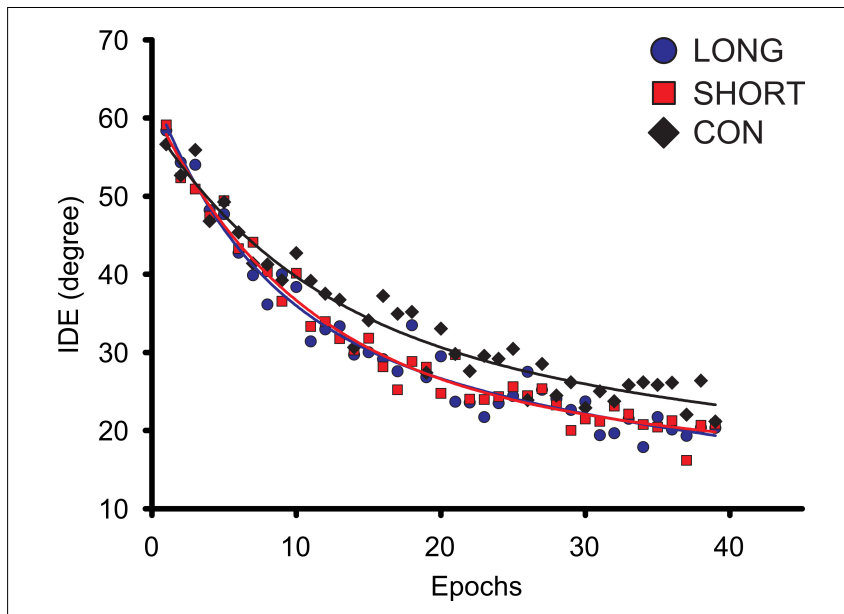

FIGURE 3 | Group average learning curves for the raw initial directional error (IDE) during the adaptation set. Each data point represents the average of eight consecutive trials (one epoch). LONG, long exercise bout before the rVMA task; SHORT, short exercise bout before the rVMA task; CON, no exercise before the rVMA task.

in children. In addition to this evidence, one should also consider exercise-increased activation of certain brain areas as a potential underlying mechanism for the benefits of exercise in relation to learning a motor task (Rajab et al., 2014; Stavrinos and Coxon, 2016). Previous evidence revealed how IE promoted a sustained increase in corticospinal excitability, which was related to the motor skill performance off-line improvements obtained during memory consolidation (Ostadan et al., 2016). Moreover, another recent study demonstrated that exercisemodulated brain activity, functional connectivity and corticomuscular coherence, improved motor memory consolidation of a motor skill when compared to a non-exercise control group (Dal Maso et al., 2018). However, at present we have no

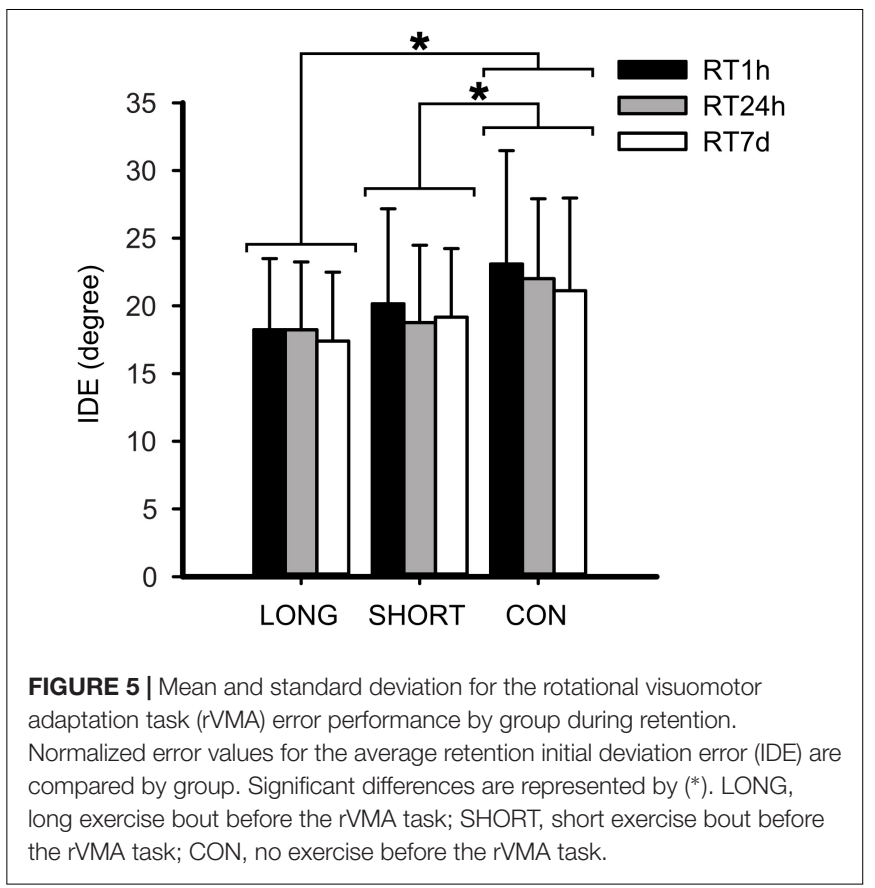

available information regarding brain activity related to exercise and motor learning in children. Therefore, further research is required to explore the potential mechanisms underlying the existing beneficial relation between exercise and motor memory consolidation, especially in children.

This study presents new insights about the influence of exercise duration on the consolidation of procedural memory in children. The results in the present study suggest that IE can enhance children's motor learning (mostly consolidation), even with bouts as short as $5 \mathrm{~min}$. Previous studies demonstrated benefits in children's attention when performing short exercise sessions during school hours (Mahar et al., 2006; Ma et al., 2014).
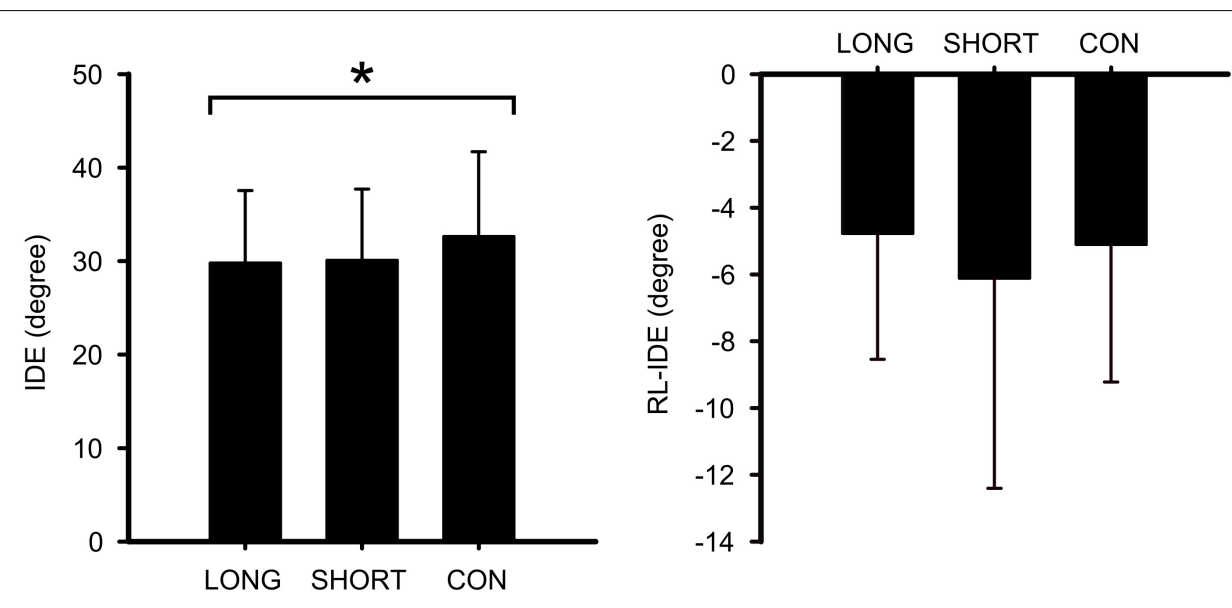

FIGURE 4 | Group average error and rate of learning during the adaptation set by group. Mean and standard deviations of the initial directional error (IDE) and the rate of learning (RL-IDE) during the adaptation set of the rotational visuomotor adaptation task (rVMA) are presented. Normalized IDE and RL-IDE values are compared among groups. A significant main group effect is represented by $\left(^{*}\right)$. LONG, long exercise bout before the rVMA task; SHORT, short exercise bout before the rVMA task; CON, no exercise before the rVMA task. 
Therefore, we think that short IE bouts could be helpful in school or sport environments, where children engage in the learning of many new motor skills. Whether similar results can be found with different types of exercise should be explored in future research, given that different exercise types yielded positive motor memory consolidation in adults (Thomas et al., 2017). The limitations of our study include: difficulties to generalize exercise benefits to other motor skills or forms of learning, missing a comparison group where exercise was performed after the adaptation set, relatively small sample, and initial differences between sites. Despite these limitations, we argue that IE with durations of 5 or 13 min could improve motor memory consolidation. Considering previous evidence regarding the moderator effect of exercise intensity, along with the present study results, one could consider the possible dose-response relation between exercise and motor learning enhancements, a topic that should be more thoroughly addressed in future research.

\section{CONCLUSION}

Retention of the rVMA was enhanced through acute exercise in children. These data suggest that motor memory consolidation was improved even when the exercise duration was only $5 \mathrm{~min}$. As expected, IE bouts did not improve the adaptation of the motor task, even when exercise duration was short. The presented exercise benefits on motor memory consolidation in children could support the use of acute IE bouts in school and sport environments.

\section{DATA AVAILABILITY}

All datasets generated for this study are included in the manuscript and/or the supplementary files.

\section{REFERENCES}

Benítez-Porres, J., López-Fernández, I., Raya, J. F., Álvarez Carnero, S., AlveroCruz, J. R., and Álvarez Carnero, E. (2016). Reliability and validity of the PAQ-C questionnaire to assess physical activity in children. J. Sch. Health 86, 677-685. doi: 10.1111 josh. 12418

Coats, R. O., Wilson, A. D., Snapp-Childs, W., Fath, A. J., and Bingham, G. P. (2014). The 50s cliff: perceptuo-motor learning rates across the lifespan. PLoS One 9:e85758. doi: 10.1371/journal.pone.0085758

Cohen, J. (1988). Statistical Power Analysis for The Behavioral Sciences. Hillsdale, NJ: Lawrence Erlbaum Associates.

Contreras-Vidal, J. L. (2006). Development of forward models for hand localization and movement control in 6- to 10-year-old children. Hum. Mov. Sci. 25, 634-645. doi: 10.1016/j.humov.2006.07.006

Contreras-Vidal, J. L., Bo, J., Boudreau, J. P., and Clark, J. E. (2005). Development of visuomotor representations for hand movement in young children. Exp. Brain Res. 162, 155-164. doi: 10.1007/s00221-004-2123-2127

Dal Maso, F., Desormeau, B., Boudrias, M. H., and Roig, M. (2018). Acute cardiovascular exercise promotes functional changes in cortico-motor networks during the early stages of motor memory consolidation. Neuroimage 174, 380-392. doi: 10.1016/j.neuroimage.2018.03.029

Etnier, J., Labban, J. D., Piepmeier, A., Davis, M. E., and Henning, D. A. (2014). Effects of an acute bout of exercise on memory in 6 th grade children. Pediatr. Exerc. Sci. 26, 250-258. doi: 10.1123/pes.2013-2141

\section{ETHICS STATEMENT}

Written consent from all participants' guardians and written assent from participants were provided prior to initiate the study in accordance with the Declaration of Helsinki. The study was approved by the Ethic Committee of Clinic Researches of the Catalan Sport Administration and the Committee for the Protection of Human Subjects of the California State University, Northridge, CA, United States.

\section{AUTHOR CONTRIBUTIONS}

All authors participated in the study conceptualization, data collection and analysis, and manuscript preparation and revision, and contributed equally to this study.

\section{FUNDING}

This study was supported by the Institut Nacional d'Educació Física de Catalunya (INEFC) from the Generalitat de Catalunya, Agència de Gestió d'Ajuts Universitaris i de Recerca (AGAUR; PRE/2623/2013), the Grup de Recerca en Activitat Física i Salut (GRAFiS, Generalitat de Catalunya 2017SGR/741) and a California State University grant.

\section{ACKNOWLEDGMENTS}

We want to thank Jennifer Romack for helping during the study preparation and multiple CSUN undergraduate students for assisting with data collection. We also want to thank all participants, families, and schools involved in this study.

Ferrer-Uris, B., Busquets, A., and Angulo-Barroso, R. (2018). Adaptation and retention of a perceptual-motor task in children: effects of a single bout of intense endurance exercise. J. Sport Exerc. Psychol. 40, 1-9. doi: 10.1123/jsep. 2017-2044

Ferrer-Uris, B., Busquets, A., Lopez-Alonso, V., Fernandez-del-Olmo, M., and Angulo-Barroso, R. (2017). Enhancing consolidation of a rotational visuomotor adaptation task through acute exercise. PLoS One 12:e0175296. doi: 10.1371/ journal.pone.0175296

Kagerer, F. A., and Clark, J. E. (2014). Development of interactions between sensorimotor representations in school-aged children. Hum. Mov. Sci. 34, 164-177. doi: 10.1016/j.humov.2014.02.001

King, B. R., Kagerer, F. A., Contreras-Vidal, J. L., and Clark, J. E. (2009). Evidence for multisensory spatial-to-motor transformations in aiming movements of children. J. Neurophysiol. 101, 315-322. doi: 10.1152/jn.90781.2008

Kowalski, K. C., Crocker, P. R. E., and Faulkner, R. A. (1997). Validation of the physical activity questionnaire for older children. Pediatr. Exerc. Sci. 9, 174-186. doi: $10.3967 /$ bes 2016.022

Krakauer, J. W., Ghez, C., and Ghilardi, M. F. (2005). Adaptation to visuomotor transformations: consolidation, interference, and forgetting. J. Neurosci. 25, 473-478. doi: 10.1523/JNEUROSCI.4218-04.2005

Krakauer, J. W., and Shadmehr, R. (2006). Consolidation of motor memory. Trends Neurosci. 29, 58-64. doi: 10.1016/j.tins.2005.10.003

Labelle, V., Bosquet, L., Mekary, S., and Bherer, L. (2013). Decline in executive control during acute bouts of exercise as a function of exercise 
intensity and fitness level. Brain Cogn. 81, 10-17. doi: 10.1016/j.bandc.2012. 10.001

Léger, L. A., Mercier, D., Gadoury, C., and Lambert, J. (1988). The multistage 20 metre shuttle run test for aerobic fitness. J. Sports Sci. 6, 93-101. doi: 10.1080/ 02640418808729800

Lundbye-Jensen, J., Skriver, K., Nielsen, J. B., and Roig, M. (2017). Acute exercise improves motor memory consolidation in preadolescent children. Front. Hum. Neurosci. 11:182. doi: 10.3389/fnhum.2017.00182

Ma, J. K., Le Mare, L., and Gurd, B. J. (2014). Classroom-based high-intensity interval activity improves off-task behaviour in primary school students. Appl. Physiol. Nutr. Metab. 39, 1332-1337. doi: 10.1139/apnm-2014-2125

Mahar, M. T., Murphy, S. K., Rowe, D. A., Golden, J., Shields, A. T., and Raedeke, T. D. (2006). Effects of a classroom-based program on physical activity and on-task behavior. Med. Sci. Sports Exerc. 38, 2086-2094. doi: 10.1249/01.mss. 0000235359.16685.a3

Mang, C. S., Brown, K. E., Neva, J. L., Snow, N. J., Campbell, K. L., and Boyd, L. A. (2016). Promoting motor cortical plasticity with acute aerobic exercise: a role for cerebellar circuits. Neural Plast. 2016:6797928. doi: 10.1155/2016/6797928

Mang, C. S., Snow, N. J., Campbell, K. L., Ross, C. J. D., and Boyd, L. A. (2014). A single bout of high-intensity aerobic exercise facilitates response to paired associative stimulation and promotes sequence-specific implicit motor learning. J. Appl. Physiol. 117, 1325-1336. doi: 10.1152/japplphysiol.00498.2014

McMorris, T., and Hale, B. J. (2012). Differential effects of differing intensities of acute exercise on speed and accuracy of cognition: a meta-analytical investigation. Brain Cogn. 80, 338-351. doi: 10.1016/j.bandc.2012.09.001

Ostadan, F., Centeno, C., Daloze, J. -F., Frenn, M., Lundbye-Jensen, J., and Roig, M. (2016). Changes in corticospinal excitability during consolidation predict acute exercise-induced off-line gains in procedural memory. Neurobiol. Learn. Mem. 136, 196-203. doi: 10.1016/j.nlm.2016.10.009

Rajab, A. S., Crane, D. E., Middleton, L. E., Robertson, A. D., Hampson, M., and MacIntosh, B. J. (2014). A single session of exercise increases connectivity in sensorimotor-related brain networks: a resting-state fMRI study in young healthy adults. Front. Hum. Neurosci. 8:625. doi: 10.3389/fnhum.2014.00625

Rhee, J., Chen, J., Riechman, S. M., Handa, A., Bhatia, S., and Wright, D. L. (2015). An acute bout of aerobic exercise can protect immediate offline motor sequence gains. Psychol. Res. 80, 518-531. doi: 10.1007/s00426-015-0682-689

Roig, M., Skriver, K., Lundbye-Jensen, J., Kiens, B., and Nielsen, J. B. (2012). A single bout of exercise improves motor memory. PLoS One 7:e44594. doi: 10.1371/journal.pone.0044594

Roig, M., Thomas, R., Mang, C. S., Snow, N. J., Ostadan, F., Boyd, L. A., et al. (2016). Time-Dependent effects of cardiovascular exercise on memory. Exerc. Sport Sci. Rev. 44, 81-88. doi: 10.1249/JES.0000000000000078

Segal, S., Cotman, C., and Cahill, L. (2012). Exercise-Induced noradrenergic activation enhances memory consolidation in both normal aging and patients with amnestic mild cognitive impairment. J. Alzheimers Dis. 32, 1011-1018. doi: 10.3233/JAD-2012-121078
Skriver, K., Roig, M., Lundbye-Jensen, J., Pingel, J., Helge, J. W., Kiens, B., et al. (2014). Acute exercise improves motor memory: exploring potential biomarkers. Neurobiol. Learn. Mem. 116, 46-58. doi: 10.1016/j.nlm.2014. 08.004

Snow, N. J., Mang, C. S., Roig, M., McDonnell, M. N., Campbell, K. L., and Boyd, L. A. (2016). The effect of an acute bout of moderate-intensity aerobic exercise on motor learning of a continuous tracking task. PLoS One 11:e0150039. doi: 10.1371/journal.pone.0150039

Statton, M. A., Encarnacion, M., Celnik, P., and Bastian, A. J. (2015). A single bout of moderate aerobic exercise improves motor skill acquisition. PLoS One 10:e0141393. doi: 10.1371/journal.pone.0141393

Stavrinos, E. L., and Coxon, J. P. (2016). High-intensity interval exercise promotes motor cortex disinhibition and early motor skill consolidation. J. Cogn. Neurosci. 26, 1-12. doi: 10.1162/jocn_a_01078

Taubert, M., Villringer, A., and Lehmann, N. (2015). Endurance exercise as an "Endogenous" neuro-enhancement strategy to facilitate motor learning. Front. Hum. Neurosci. 9:692. doi: 10.3389/fnhum.2015.00692

Thomas, R., Beck, M. M., Lind, R. R., Korsgaard Johnsen, L., Geertsen, S. S., Christiansen, L., et al. (2016a). Acute exercise and motor memory consolidation: the role of exercise timing. Neural Plast. 2016, 1-16. doi: 10.1155/ 2016/6205452

Thomas, R., Johnsen, L. K., Geertsen, S. S., Christiansen, L., Ritz, C., Roig, M., et al. (2016b). Acute exercise and motor memory consolidation: the role of exercise intensity. PLoS One 11:e0159589. doi: 10.1371/journal.pone.0159589

Thomas, R., Flindtgaard, M., Skriver, K., Geertsen, S. S., Christiansen, L., Korsgaard Johnsen, L., et al. (2017). Acute exercise and motor memory consolidation: does exercise type play a role? Scand. J. Med. Sci. Sport 27, 1523-1532. doi: $10.1111 /$ sms.12791

Wigal, S. B., Nemet, D., Swanson, J. M., Regino, R., Trampush, J., Ziegler, M. G., et al. (2003). Catecholamine response to exercise in children with attention deficit hyperactivity disorder. Pediatr. Res. 53, 756-761. doi: 10.1203/01.PDR. 0000061750.71168 .23

Winter, B., Breitenstein, C., Mooren, F. C., Voelker, K., Fobker, M., Lechtermann, A., et al. (2007). High impact running improves learning. Neurobiol. Learn. Mem. 87, 597-609. doi: 10.1016/j.nlm.2006.11.003

Conflict of Interest Statement: The authors declare that the research was conducted in the absence of any commercial or financial relationships that could be construed as a potential conflict of interest.

Copyright (c) 2019 Angulo-Barroso, Ferrer-Uris and Busquets. This is an open-access article distributed under the terms of the Creative Commons Attribution License (CC BY). The use, distribution or reproduction in other forums is permitted, provided the original author(s) and the copyright owner(s) are credited and that the original publication in this journal is cited, in accordance with accepted academic practice. No use, distribution or reproduction is permitted which does not comply with these terms. 